\title{
Presentación: más allá de la información
}

Presentation: beyond information

\section{Francisco Javier GARCíA MARCo}

\author{
Universidad de Zaragoza, Facultad de Filosofía y Letras (España), jgarcia@unizar.es
}

\begin{abstract}
Resumen
Se presentan los artículos que componen el número 1 del volumen 17 de la revista Scire: Organización y Representación del Conocimiento (en.-jun. 2011). Se abordan la calidad de la información, las relaciones entre las disciplinas de la información y el conocimiento, el orden de cita en las clasificaciones de niveles integradores, los avances en la ISBD, la detección de cuasi-duplicados, la superioridad de la recuperación con vocabularios controlados en contextos especializados, las implicaciones de la diplomática para la representación del conocimiento archivístico, el papel de la clasificación en el OPAC, la construcción de la organización del conocimiento como disciplina científica, y los recursos de información en las empresas manufactureras. Todos los artículos resaltan de una manera u otra la inevitabilidad del problema del conocimiento, y la gestión de la información como trabajo de mediación, que, lejos de limitarse a tareas técnicas y administrativas, tiene un importantísimo papel de creación de nuevas relaciones en los entornos reales de información y comunicación.
\end{abstract}

Palabras clave: Organización del conocimiento. Análisis de contenido. Teoría. Aplicaciones.

Componen este número un conjunto muy interesante de trabajos que abordan tanto perspectivas teóricas y estratégicas como temas y proyectos específicos. Juntos constituyen un buen ejemplo de las tendencias actuales en la representación, organización y gestión del conocimiento, y reúnen la reflexión teórica y social con la investigación experimental, la indagación cualitativa con la cuantitativa.

En este mundo de información desbordada, desbordante y muchas veces redundante que ha traído la Web, Peter Ohly (2011) se pregunta precisamente por el significado de la calidad de la información, por cómo se puede asegurar y por el papel de los científicos de la información en esta misión. Su punto de partida es que no hay una correspondencia biunívoca, dada para siempre, entre la información y sus usos; al contrario, esa relación es susceptible de permanente cambio y creación. La calidad es una función del uso de la información. Por otra parte, además de respetando las cualidades instrínse-

\begin{abstract}
The articles published in the number 1 of the volume 17th of Scire (Jan.-June 2011) are presented. This issue includes articles on information quality, the ecology of the information and knowledge disciplines, citation order in integrative levels classifications, ISBD advances in the representation of digital records, near-duplicates detection, the superiority of controlled retrieval in specialized contexts, the implications of Diplomatics for the representation of knowledge in archives, the role of classification in the OPAC, the building of knowledge organization as a scientific discipline, and the use of information in manufacturing organizations. All these papers highlight in different ways that information science cannot dismiss the problem of knowledge, and that information management is a mediation work that is not reduced to administrative and technical tasks, but includes also the creation and development of new relations in the real environments where people get informed and communicate.
\end{abstract}

Keywords: Knowledge organization. Content analysis. Theory. Applications.

cas de la información —verdad y alcance-, es posible mejorar su uso mediante diversas estrategias que aprovechan las características de la información que están ligadas a los diferentes momentos de su ciclo de vida: su creación, procesamiento, recuperación y uso. Una de las observaciones más agudas del autor es que, a priori, las soluciones no suelen ser sumativas: una estrategia que favorece unos aspectos puede perjudicar en otros:

e.g. a user driven system might be difficult to be enhanced by semantic or knowledge based techniques; user friendly presentation and sophisticated retrieval and mining techniques seem to be opposite positions.

$\mathrm{Y}$, sin embargo, una vez reconocidas las ventajas e inconvenientes de cada aproximación, es posible combinarlas de forma inteligente.

Quality is thus not static nor relative, but a question of adaptability to user groups, user needs, and user expectations. 
En la búsqueda de la calidad, Alan Gilchrist (2011) se centra en la de los productos de organización del conocimiento, uno de los puentes fundamentales que se han inventado para comunicar los documentos con sus usuarios. De forma semejante a Peter Ohly, Alan Gilchrist propone una mayor colaboración entre las perspectivas alternativas, en este caso entre los profesionales de la organización del conocimiento y los de la recuperación de la información. El autor hace una cuidadosa distinción del alcance real entre las diferentes disciplinas de la información y el conocimiento -y entre estos mismos conceptos- con una claridad que solo puede proporcionar su amplísima perspectiva. Alan Gilchrist muestra claramente que los profesionales de la información la gestionan sin centrarse en su grado de verdad -ese juicio, que queda para el usuario-, sino en la correspondencia temática entre la oferta y la demanda, indagada de formas diversas.

En el artículo de Alan Gilchrist, la organización del conocimiento aparece también como trabajo de mediación, una mediación que requiere primero levantar el mapa del espacio del problema. Con su habitual agudeza reseña a Lambe (in press) para definir con precisión la oportunidad que el momento ofrece a la organización del conocimiento:

[...] in the current complex state of scientific research, involving multidisciplinary teams, KO had a role to play in identifying and recording new boundaries and overlaps between apparently disparate subject areas as an aid to sense-making in the pursuit of scientific discovery.

En este contexto, el acercamiento entre los profesionales de la recuperación de información y de la organización del conocimiento es todavía más necesario, y debe discurrir en ambas direcciones. La organización del conocimiento encuentra su principal aplicación en la recuperación de información; y la recuperación de la información no puede limitarse tan solo a las palabras, pues éstas son al final útiles por el significado que portan o permiten construir, y ese significado está siempre en re-relación y construcción:

Information is also abstract and information scientists must work with 'messages' which are the attempts of knowledge creators to disseminate their opinions. [...] The eminent information scientist Robert Fairthorne once said "It is not the job of information scientists to give information, but information about information". One might add that it is not the job of those concerned with $\mathrm{KO}$ to give knowledge, but information about knowledge; and this is achieved through the construction of models.
Claudio Gnoli (2011) presenta una adaptación a la teoría de la clasifiación de niveles integradores - que es el modelo que sustenta a las clasificaciones universales - para dar cuenta de las necesidades que plantea un entorno científico cada vez más interdisciplinar, en el que el foco está menos en la disciplina y más en los objetos de la realidad. Es un trabajo importante en el que, además de aportar una solución al problema técnico del orden de cita en contextos interdisciplinares, proporciona una síntesis actualizada de su modelo teórico del campo de la organización del conocimiento:

Knowledge carried by one document usually contains elements of all these dimensions: it deals with some set of phenomena (ontology) under a certain perspective (epistemology) recorded in some document form (bibliography) kept with other documents in a collection (library science, museology etc.) for certain categories of users (sociology).

A la hora de sistematizar el conocimiento hay que optar entre la perspectiva epistemológica, ontológica o pragmática; $y$, dado que cualquier compromiso con una sacrifica a las demás, es necesario tender puentes entre sistemas alternativos, lo que es posible hoy en día gracias a los estándares que promueven la interoperabilidad y a proyectos como la Integrative Levels Classification.

A continuación Ana Belén Ríos Hilario (2011) presenta un análisis del tratamiento de los recursos electrónicos en la ISBD Consolidada y lo encuadra dentro del largo movimiento de reforma de las reglas de catalogación, que responde a la aparición y creciente importancia de los documentos no librarios, movimiento que culmina con la Internet y los recursos remotos. La propia edición de la ISBD Consolidada resulta ser un buen ejemplo de que no existen normalmente respuestas únicas en documentación, sino soluciones adaptadas a diferentes tipos de necesidades y usuarios: La ISBD Consolidada permite dar coherencia al cuerpo de conocimiento, pero dificulta su consulta para cada tipo de material específico. Los arquitectos de las ISBD necesitan un documento máster; los catalogadores, resúmenes adaptados a sus necesidades, que serán creados a partir del primero.

Carlos G. Figuerola, Raquel Gómez Díaz, José L. Alonso Berrocal y Ángel F. Zazo Rodríguez (2011) presentan un trabajo de investigación experimental sobre un aspecto crucial de la información contemporánea que está relacionado con el problema de la calidad de información: la redundancia de información, que centran en el problema más específico, pero tremendamente relevante, de la detección de casi-duplicados. La redundancia de información no detectada es 
fuente de una pérdida considerable de tiempo, y gran parte de esas duplicaciones pasan desapercibidas porque no son exactas. Para resolver este problema aplican técnicas de fuzzy hashing con excelentes resultados en una dirección muy innovadora.

Vera Regina Casari Boccato, Mariângela Spotti Lopes Fujita e Isidoro Gil Leiva (2011) presentan también una investigación empírica sobre la recuperación de materias en catálogos bibliográficos de bibliotecas universitarias por usuarios finales, que prueba una vez más la superioridad de la recuperación apoyada en vocabularios controlados sobre la recuperación en lenguaje natural en entornos especializados. En ellos el lenguaje natural queda gravemente afectado por su baja precisión, característica que entra en colisión directa con la especificidad de las necesidades de información de los usuarios típicos de una biblioteca universitaria, que requieren de información especializada. Además, muestran como estos dominios específicos de conocimiento son mejor servidos por vocabularios de recuperación especialmente diseñados y mantenidos para ellos.

Sonia Troitiño (2011) investiga las diferencias entre los modelos de análisis documental empleados por la diplomática tradicional y la tipología documental, dos de las fuentes más importantes de la descripción archivística. Es un hito señalado dentro de la línea de investigación que dirige en Marilia el profesor José Augusto Chaves Guimarães sobre las raíces del análisis documental de contenido en las diferentes disciplinas documentales. La combinación de ambas técnicas permite una suerte de arqueología documental y una inmersión en la larga duración de los tipos documentales, poniendo a la empiria documental al servicio del estudio de las grandes tendencias de la historia humana, reflejadas en la evolución de los tipos documentales.

Ana María Martínez Tamayo, Leslie Bava y María Inés Kessler (2011) presentan un estudio muy interesante, en este caso de carácter empírico, sobre la funcionalidad que proporcionan los catálogos en línea de las bibliotecas universitarias argentinas para aprovechar todas las posibilidades de las clasificaciones bibliográficas en la recuperación y navegación de los recursos bibliográficos. Con este estudio de alcance nacional contribuyen a demostrar que las ventajas de la clasificación para la recuperación de información están desaprovechadas en la mayoría de los catálogos, aunque también reseñan las tendencias más importantes en la actualidad para poner en valor este recurso fundamental, al que tanto tiempo se dedica en muchas bibliotecas.
Seguidamente, Rodrigo Rabello (2011) nos presenta una indagación teórica sobre la organización del conocimiento desde la perspectiva bachelriana de la construcción de objetos científicos. Es un interesante trabajo de filosofía de la ciencia en el que se analizan la organización del conocimiento y la ciencia de la información como disciplinas científicas $-\mathrm{y}$, por tanto, estructuras socio-epistemológicas sujetas a un permanente proceso de cambio y ajuste, en ocasiones incluso revolucionario. Rodrigo Rabello muestra como son los procesos del ciclo de la información los que elicitan la formación de disciplinas dentro de la ciencia de la información, más que el propio concepto de información; lo que es esperable en una disciplina de clara orientación técnica y prágmática. No es extraño que la reflexión sobre la propia información se esté configurando actualmente más como una "disciplina interdisciplinar" - the Science of Information-, en la que la contribución de la Information Science es solo una más.

Finalmente, Celso Martínez Musiño (2011) aborda un tema poco tratado, a saber, el uso de la información en las industrias manufactureras. En el sector productivo muchas veces la información fluye por canales no escritos, a través de instrucciones, cursos y fundamentalmente la opinión de los expertos y consultores. Este hecho ha provocado posiblemente que se perciba como un nicho en el que hay poco lugar para los profesionales de la información, con algunas notables excepciones. Celso Martínez muestra diversos proyectos de investigación que han demostrado que estas organizaciones también pueden beneficiarse de la gestión de la información, y aborda una clasificación de los recursos de información que más demandan y utilizan, y que, por tanto, son susceptibles de constituir el núcleo de la actividad informacional. La conclusión más importante, y una interesante anomalía teórica desde el punto de vista de la ciencia de la información, es que las personas son una fuente de información clave en las empresas manufactureras, como, por otra parte, ocurre en una gran parte de las industrias productivas y servicios. Es normal, por tanto que la gestión de la información tenga que ir de la mano de otras disciplinas al entrar en este campo tan necesitado y prometedor, en particular de la gestión del conocimiento, y en estrecha colaboración con los departamentos de dirección, marketing y recursos humanos.

En fin, la ciencia de la información -en sentido amplio, no como la unión de recuperación de la información y técnicas bibliométricas - dista de ser una ciencia en el sentido tradicional de la palabra; porque, aunque se reconoce en ella un 
dominio específico -esto es, la comprensión del fenómeno de la información humana y la mejora de su transferencia mediante el desarrollo y aplicación de modelos y tecnologías-, no posee un único ejemplar compartido en el sentido kuhniano de la palabra. Y, sin embargo, la invención de la World Wide Web, con su gran potencial de integración de medios y tecnologías, y los esfuerzos diversos y procedentes de distintas disciplinas por transferir a ella el conjunto de la información está contribuyendo a acercar ambos polos de forma eficaz.

Por otra parte, la información como profesión es un campo cada vez más prometedor, siempre que se entienda como trabajo y servicio. La información es un bien, y como tal debe ser útil a sus usuarios específicos. La labor de los mediadores es acercar la oferta y la demanda actual y potencial, y en la medida que lo consigan añadirán más valor, y obtendrán una relevancia y una recompensa mayor. Al final, la calidad de la información y de su transferencia mercadeo- es un trabajo, que, aunque muchas veces se ve reducido a tareas administrativas de control de calidad que cada vez resultan más automatizables, también incluye la detección y desarrollo de usos potenciales y, por cierto, la invención de nuevos usos y procedimientos.

\section{Referencias}

Boccato, Vera Regina C.; Fujita, Mariângela Spotti L.; Gil Leiva, Isidoro (2011). Avaliação comparada do uso de linguagens de indexação em catálogos de bibliotecas universitárias para recuperação por assunto // Scire. $17: 1$ (en.-jun. 2011) 55-64
Eito Brun, Ricardo (2011). Aplicabilidad de EAD en archivos históricos de ingeniería: el fondo Carlos Fernández Casado. // Scire. 17:1 (en.-jun. 2011) 35-39.

Figuerola, Carlos G.; Gómez Díaz, Raquel; Alonso Berrocal, José Luis; Zazo Rodríguez, Ángel Francisco (2011). Documentos duplicados y casi duplicados en el Web: detección con técnicas de hashing borroso. // Scire. 17:1 (en.-jun. 2011) 49-54.

Gilchrist, Alan (2011). A note on the theory and practice of knowledge organization and knowledge representation. // Scire. 17:1 (en.-jun. 2011) 23-28.

Gnoli, Claudio (2011). Ontological foundations in knowledge organization: the theory of integrative levels applied in citation order // Scire. 17:1 (en.-jun. 2011) 29-34.

Lambe, Patrick (In press). Knowledge organization systems as enablers to the conduct of science. // Gilchrist, A.; Vernau, Judi (Eds). Facets of Knowledge Organization: proceedings of the ISKO(UK) Biennial Conference, 4th/5th July 2011, London. Bingley: Emerald Group Publishing.

Martínez Musiño, Celso (2011). Tipificación y uso de los recursos de información en las organizaciones manufactureras. // Scire. 17:1 (en.-jun. 2011) 93-100.

Martínez Tamayo, Ana María; Bava, Leslie; Kessler, María Inés (2011). Las funciones de la clasificación en los catálogos en línea de bibliotecas universitarias argentinas. // Scire. 17:1 (en.-jun. 2011) 73-79.

Ohly, H. Peter (2011). Information: a question of quality? // Scire. 17:1 (en.-jun. 2011) 17-21.

Rabello, Rodrigo (2011). La construcción de objetos en la Ciencia de la Información: el caso del proceso organización del conocimiento. // Scire. 17:1 (en.-jun. 2011) 81-91

Ríos Hilario, Ana Belén; Guerreiro, João (2011). La aplicación de la ISBD consolidada en la descripción de los recursos electrónicos: similitudes y diferencias con la ISBD (ER). // Scire. 17:1 (en.-jun. 2011) 41-48.

Rodríguez, Sonia Troitiño (2011). Da crítica diplomática à análise tipológica: abordagens e técnicas de análise. // Scire. 17:1 (en.-jun. 2011) 65-72. 